\title{
Manual Lymphatic Drainage Therapy in the Knee Joint Functional Rehabilitation After TKA in Diabetic Knee Osteoarthritis Patients: A Randomized Clinical Trial
}

\author{
Hong Zhang, Jiajie Yan, Sujuan Lin, Huajun Wang*, Lei Xiao, Guorong She, Ning Liu, \\ Zhengang Zha*
}

Department of Orthopedics, the First Affiliated Hospital, Jinan University, Guangzhou, China

Email address:

zhzgg@vip.163.com (Zhengang Zha),whj323@126.com (Huajun Wang)

${ }^{*}$ Corresponding author

\section{To cite this article:}

Hong Zhang, Jiajie Yan, Sujuan Lin, Huajun Wang, Lei Xiao, Guorong She, Ning Liu, Zhengang Zha. Manual Lymphatic Drainage Therapy in the Knee Joint Functional Rehabilitation After TKA in Diabetic Knee Osteoarthritis Patients: A Randomized Clinical Trial. Journal of Surgery. Vol. 7, No. 3, 2019, pp. 50-56. doi: 10.11648/j.js.20190703.11

Received: April 3, 2019; Accepted: May 16, 2019; Published: May 30, 2019

\begin{abstract}
To investigate the clinical effect of manual lymphatic drainage on functional rehabilitation of patients with diabetic knee osteoarthritis. A total of 199 patients with diabetic osteoarthritis who underwent total knee arthroplasty (TKA) were enrolled in the hospital and there were 72 males and 127 females in these patients. They were randomly divided into manual lymphatic drainage group (103 cases) and routine care group (96 cases). The manual lymphatic drainage group was treated with manual lymphatic drainage on the basis of routine functional rehabilitation nursing in March, while the routine nursing group only received perioperative nursing for conventional knee replacement. The patients with manual lymphatic drainage and routine nursing were studied before and 1 week, 1 month, 1 month, 3 months, and 6 months after operation to get the results of the changes of knee active flexion and extension, limb swelling degree, VAS pain score and preoperative group, the difference of HSS score and AKS score of knee joint in 6 months after operation. The active knee flexion activity of patients in the manual lymphatic drainage group was better than that of the routine care group $(P<0.05)$. The active knee extension and knee swelling were better in the manual lymphatic drainage group than that in the routine nursing group 1 week and 1 month after operation $(P$ $<0.05)$; there was no significant difference in knee active activity and knee swelling between the two groups in March and June $(P>0.05)$; The scores of HSS in patients with manual lymphatic drainage in each stage after operation were better than that in the routine care group $(P<0.05)$. The AKS pain scores of the manual lymphatic drainage group were better than those of the routine care group $(P<0.05)$. There was no statistical difference $(P>0.05)$ in the AKS pain scores of the two groups in March and June. The AKS clinical score and AKS function score of patients in the manual lymphatic drainage group were better than that in the routine care group $(P<0.05)$. In general, manual lymphatic drainage treatment for patients with diabetes after total knee surgery can not only effectively alleviate postoperative knee swelling and pain but can also accelerate knee joint function recovery and improve postoperative satisfaction.
\end{abstract}

Keywords: Manual Lymphatic Drainage, Total Knee Replacement, Diabetic Patients, Functional Rehabilitation, Clinical Efficacy

\section{Introduction}

It is well-known that the main pathological changes of osteoarthritis are degenerative lesions of cartilage and secondary bone hyperplasia [1]. The data shows that the number of patients with osteoarthritis exceeds $10 \%$ of the population, and more than 100 million in China. This phenomenon can occur in any joint of the human body, most common in the knee joint $[2,3]$. Moreover, the incidence of knee osteoarthritis in China increased by $45 \%$ between 1990 and 2010. Knee osteoarthritis is also one of the main causes of disability, and its final disability rate is $21.3 \%$. It has been estimated that the cost of treatment for chronic diseases in orthopedics in China will increase to 85 billion by 2020 and will 
reach 1.8 trillion yuan by 2050 . So, knee osteoarthritis will not only seriously endanger the health of patients but will also bring great economic burden to the society. Since total knee arthroplasty (TKA) can effectively relieve pain and restore knee function, so it is the best way to treat knee osteoarthritis caused by various causes, which is regarded as the most successful operation in the 21st century [4-6]. However, it is reported that the patient who are satisfied after TKA is still only $81 \%$ to $89 \%$. In order to maximize the recovery of knee function, we should also consider postoperative scientific, systematic, safe and effective rehabilitation in addition to accurate surgical options, especially for elderly patients with complications such as diabetes. At present, China has become the country with the most diabetes patients in the world. In 2013, there were 98 million people with diabetes in the country and it is expected to increase to 143 million by 2035. With the aging of the population, there will be more and more knee osteoarthritis patients with diabetes in the future. In recent years, studies have shown that diabetes is an independent risk factor for the development [7, 8]. Moreover, diabetes directly or indirectly participates and induces the formation and development of knee osteoarthritis. Diabetic patients often have peripheral vascular neuropathy which can often lead to postoperative pain, swelling, wound and periprosthetic infection, postoperative sacral fracture, prosthesis loosening and other postoperative complications after total knee arthroplasty, so the rehabilitation of patients with diabetic knee osteoarthritis after TKA is more urgent to solve. MLD (Manual Lymphatic Drainage) $[9,10]$ is a lymphatic drainage detoxification technique which is mainly to push down the lymphatic flow. It is a technique for excreting body fluid toxins. MLD is widely used in developed countries such as Germany and the United States. In China, MLD has been applied to upper extremity edema after breast cancer surgery, lower extremity edema after cervical cancer surgery, and has achieved good results, however lymphatic massage has little clinical research on functional rehabilitation after TKA in diabetic patients. In this study, we have analyzed the effect of MLD for patients with diabetic knee osteoarthritis after total knee surgery. On the one hand, we have detected the knee proactive flexion and extension, the degree of swelling of the affected limb, and the degree of swelling of the affected limb before and 1 week, 1 month, 3 months, and 6 months after surgery. On the other hand, we have also detected the changes of VAS pain scores and the changes of knee joint HSS scores and AKS scores before and after 1 month, 3 months and 6 months in the two groups. The clinical effect of MLD on functional rehabilitation after TKA in diabetic patients was discussed.

\section{Materials and Methods}

\subsection{General Information}

A total of 199 patients with diabetic knee osteoarthritis after unilateral total knee arthroplasty were enrolled in our hospital, including 72 males and 127 females with an average age of $66.2 \pm 10.2(56-85)$ years old. These patients were divided into manual lymphatic drainage group (group M: 103 cases) and routine care group (group R: 96 cases) according to whether manual lymphatic drainage was used. The electro acupuncture treatment group was treated with manual lymphatic drainage + functional rehabilitation, and the routine care group was treated with functional rehabilitation. There were no significant differences in the basic data of age, sex, and disease between the two groups, as shown in Table 1. The study was approved by the Ethics Committee of our hospital, and all patients have signed informed consent before participating in the study.

\subsection{Inclusion Criteria and Exclusion Criteria}

Inclusion criteria: (1) The patients should be diagnosed with knee joint OA according to the OA diagnosis and treatment guidelines formulated by the Chinese Medical Association orthopedics branch; (2) The first knee TKA was performed; (3) The patients should have the type 2 diabetes for more than 10 years.

Exclusion criteria: (1) Simultaneous or staged replacement of both knee joints; (2) Patients with total knee arthroplasty also have other diseases such as traumatic osteoarthritis and rheumatoid arthritis; (3) Patients with the history of malignant tumors, hemorrhagic diseases, vascular embolism or abnormal blood coagulation mechanism; (4) Patients who have long-term use of analgesics which might influence the evaluation results of neuromuscular system diseases and the results of the experiment; (5) Patient who have severe organic disease and are intolerable Rehabilitation; (6) Mental patients.

\subsection{Surgical Methods and Routine Postoperative Treatment}

All operations were not only performed by the same group of senior physicians but also treated with combined spinal and epidural anesthesia. The affected limb should have an inflatable tourniquet with a pressure of $250 \mathrm{mmHg}$. The patient leaned back on his knees, the median incision in front of the knee, and the medial approach to the ankle. Then we removed some of the underarm fat pad, valgus the tibia, and exposed the field of view. Moreover, we positioned outside the bone marrow and in the femoral bone, then osteotomy and reaming were implemented. The cancellous bone was washed with a pulse flushing gun and the appropriate tibial prosthesis. Here we selected femoral prosthesis (BIOMET, USA) for installation and fixation. The opening of the medullary cavity was closed by the autologous bone block retained during osteotomy. Then we corrected the humerus and sutured the joint capsule. The incision was closed layer by layer, the tourniquet was loosened, and the cotton pad was pressure-wrapped, the drainage tube was placed, $50 \mathrm{ml}$ of tranexamic acid physiological saline solution was injected into the joint capsule. At last the drainage tube was clamped for 4 hours.

\subsection{Functional Rehabilitation Training Method}

(1) 1 to 2 days after surgery: long contractions of the surgical quadriceps and hamstrings, sputum pump exercises; raise the 
affected limbs at rest. (2) Day 2 postoperatively: Remove the drainage tube and continue the above exercises. (3) 3 days after surgery: the straight leg raising exercise in the supine position, the active knee flexion and extension training of the affected side knee joint, and the practice of standing and balancing exercises with the support of the lower standing. (4) 1 week after surgery: increase the active joint activity training, practice the crutches or walking on the walker; use the device to resists the isotonic contraction exercises of the quadriceps and hamstrings, and practice ascending and descending as appropriate. (6) Continue to straight leg elevation after discharge, quadriceps, hamstring muscle strength and joint mobility training.

\subsection{Manual Lymphatic Drainage Treatment}

Manual lymphatic drainage treatment was completed under the guidance of the rehabilitation doctors of our hospital. The patient was placed in the supine position and the affected limb was slightly elevated in the outreach position. Then we palpated the lymphatic tube around the knee joint of the surgical side which was usually slightly hard and distributed in a short strip shape. The tenderness was very obvious in this place. Moreover, we use middle finger to softly press each of the above lymphatic vessels for about 3 to 5 minutes. Then, the centripetal massage was performed for 20 minutes from the distal end of the surgical side knee to the proximal end. We did these exercises from the first day after surgery to the end of 3 months after surgery, twice a day.

\subsection{Evaluation Indicators}

We tested the indicators before surgery and 1 week, 1 month, 3 months, and 6 months after surgery. These evaluation indicators include the knee proactive flexion and extension, the degree of swelling of the affected limb, the VAS pain score, the knee HSS score, and the AKS score. These evaluation indicators were all completed by the same researcher.

1) Active range of motion of knee joint (AROM): We measured the data by 3 bony markers (large femur, lateral femoral condyle, external malleolus), then we recorded the data for 3 times and took the maximum value.

2) Swelling volume after knee surgery: When we measured this data, we should guarantee the knee joint to maintain a flexion of $20^{\circ}$.And the circumference of the knee joint passing through the midpoint of the humerus should be recorded 3 times, and we took the minimum value.

3) Visual analogue scale (VAS): 0 points: no pain; 3 points or less: slight pain, can endure; 4 points -6 points: pain affects sleep, still tolerable; 7 points - 10 points: Gradually strong pain, unbearable.

4) Hospital for special surgery knee score (HSS): This score was 100 points in total and the main content was 7 items: a.30 points for pain score; b. 22 points for knee function score; c. 18 points for activity score; d. 10 points for muscle strength score; e. 10 points for deformity score; $f$. 10 points for stability score. The above scores were added to obtain a preliminary total score, on the basis of which the score of the functional decline was subtracted.

5) American knee society score (AKS): The score included a knee joint clinical score of 100 points (including a pain score of 50 points) and a knee function score of 100 points.

\subsection{Statistical Analysis}

The data obtained in this study were statistically processed by SPSS13.0 software. The measurement data of the two groups of age and the normal distribution before and after treatment were measured by mean \pm standard deviation $( \pm$ SD) and compared by $\mathrm{t}$ test. Measurement data that did not conform to the normal distribution were statistically analyzed using the rank sum test. $\mathrm{P}<0.05$ was considered statistically significant.

\section{Results}

\subsection{Comparison of Active Knee Activity in Two Groups of Patients}

There was no significant difference in knee active activity between the two groups before operation (Table 1, $P>0.05$ ). The AROM in the manual lymphatic drainage group (group M) was better than that in the routine care group (group R) at 1 week, 1 month, 3 months, and 6 months after surgery. Statistical significance $(P<0.05)$; There was no significant difference in AROM between the two groups before and after surgery $(P>$ 0.05). The AROM in the group $M$ was superior to the group $\mathrm{R}$ at 1 week and 1 month after operation and the difference was statistically significant $(P<0.05)$, as shown in Table 2 .

Table 1. Comparison of data between group $M$ and group $R$.

\begin{tabular}{|c|c|c|c|c|c|}
\hline Group & $\mathbf{N}$ & $\operatorname{Sex}(M / F)$ & $\operatorname{Part}(\mathrm{L} / \mathrm{R})$ & BMI $\left(\mathrm{kg} / \mathrm{m}^{2}\right)$ & Cours (year) \\
\hline Group M & 103 & $37 / 66$ & $58 / 45$ & $27.2 \pm 5.1$ & $9.34 \pm 1.35$ \\
\hline Group R & 96 & $35 / 61$ & $54 / 42$ & $26.7 \pm 6.3$ & $10.32 \pm 1.52$ \\
\hline
\end{tabular}

Table 2. Comparison of AROM between the two groups.

\begin{tabular}{lllllll}
\hline & Group & Preoperative & 1 week & 1month & 3 months \\
\hline \multirow{2}{*}{ Flexion } & Group M & $124.04 \pm 9.91$ & $86.33 \pm 15.14$ & $95.92 \pm 13.22$ & $106.35 \pm 7.36$ \\
& Group R & $127.31 \pm 10.34$ & $81.62 \pm 16.10$ & $89.58 \pm 13.24$ & $98.82 \pm 5.57$ \\
\multirow{2}{*}{ Straighten } & Group M & $4.94 \pm 3.38$ & $3.89 \pm 3.15$ & $3.22 \pm 1.39$ & $118.95 \pm 8.89$ \\
& Group R & $5.33 \pm 4.92$ & $4.50 \pm 3.41$ & $3.85 \pm 3.88$ & $2.56 \pm 2.36$ & $2.76 \pm 2.44$ \\
\hline
\end{tabular}




\subsection{Comparison of Swelling Degree After Knee Surgery in Two Groups of Patients}

There was no significant difference in the degree of postoperative knee swelling between the two groups before and after the operation in March and June $(P>0.05)$. One week and one month after surgery, the degree of swelling of the knee in the group $\mathrm{M}$ was better than that in the group R, and the difference was statistically significant $(P<0.05)$, as shown in Table 3 .

Table 3. Comparison of swelling degree after knee surgery in both groups.

\begin{tabular}{lllll}
\hline group & Preoperative & 1 week & 1 month & 3 months \\
\hline Group M & $41.40 \pm 2.51$ & $46.34 \pm 2.23$ & $44.08 \pm 3.15$ & $43.63 \pm 2.53$ \\
Group R & $40.61 \pm 2.94$ & $51.11 \pm 3.03$ & $49.21 \pm 1.67$ & $41.86 \pm 2.22$ \\
\hline
\end{tabular}

\subsection{Comparison of VAS Pain Scores Between the Two Groups}

There was no significant difference in VAS pain scores between the two groups before and after surgery $(P>0.05)$. The VAS pain scores of the group $\mathrm{M}$ were better than the group $\mathrm{R}$ at 1 week, 1 month, and 3 months after surgery, and the difference was statistically significant $(P<0.05)$. See Table 4 .

Table 4. Comparison of VAS pain scores between the two groups.

\begin{tabular}{llllr}
\hline Group & Preoperative & 1 week & 1 month & 3 months \\
\hline Group M & $7.46 \pm 2.53$ & $6.34 \pm 2.23$ & $5.21 \pm 1.35$ & $4.13 \pm 1.23$ \\
Group R & $7.84 \pm 2.74$ & $7.11 \pm 2.63$ & $6.21 \pm 1.67$ & $3.46 \pm 0.87$ \\
\hline
\end{tabular}

\subsection{Comparison of Knee HSS Scores Between the Two Groups}

The HSS scores of the two groups were significantly improved compared with those before surgery in January, March and June. The HSS scores of the patients in the group M were better than those in the group R in January, March and June. The difference is statistically different $(P<0.05)$, see Table 5 .

Table 5. Comparison of HSS scores between the two groups.

\begin{tabular}{llll}
\hline Group & Preoperative & 1 month & 3 months \\
\hline Group M & $58.80 \pm 6.52$ & $76.12 \pm 4.53$ & $85.34 \pm 6.33$ \\
Group R & $54.35 \pm 7.36$ & $71.45 \pm 7.12$ & $80.75 \pm 5.49$ \\
\hline
\end{tabular}

\subsection{Comparison of Knee AKS Scores Between the Two Groups}

The knee AKS scores of the two groups were significantly improved at the first, third, and sixth months after surgery. There was no significant difference in the AKS pain score between the two groups at 3 and 6 months after surgery $(P>$
0.05). The AKS pain score of the group $M$ was better than that of the group $\mathrm{R}$ in the 1 month postoperatively. The difference was statistically significant $(P<0.05)$. The AKS clinical score of the group $\mathrm{M}$ in the 1st, 3rd, and 6th postoperative days and the AKS function score were all superior to the group R, and the difference was statistically significant $(P<0.05)$, as shown in Table 6.

Table 6. Comparison of knee AKS scores between the two groups.

\begin{tabular}{lllll}
\hline Rating item & Group & Preoperative & 1 month & 3 months \\
\hline \multirow{2}{*}{ AKS pain score } & Group M & $21.75 \pm 5.98$ & $37.74 \pm 6.33$ & $41.24 \pm 8.43$ \\
& Group R & $23.86 \pm 6.13$ & $32.48 \pm 7.94$ & $42.23 \pm 7.34$ \\
AKS clinical score & Group M & $67.46 \pm 8.32$ & $74.74 \pm 8.38$ & $48.54 \pm 11.72$ \\
& Group R & $68.83 \pm 6.34$ & $70.63 \pm 9.43$ & $47.27 \pm 13.53$ \\
AKS function score & Group M & $54.74 \pm 8.84$ & $78.48 \pm 9.29$ & $84.74 \pm 9.45$ \\
& Group R & $52.75 \pm 7.57$ & $72.43 \pm 7.93$ & $89.54 \pm 9.57$ \\
\hline
\end{tabular}

\section{Discussion}

Knee osteoarthritis [11] (KOA) is a degenerative osteoarthritis of the knee, characterized by articular cartilage degeneration and osteosclerosis and hyperplasia. In Europe and the United States, KOA is the main cause of the fourth female and male eighth labor loss. It is estimated that there are 50 million KOA patients in the United States, and more than $5 \%$ of retirees are forced to retire each year because of their illness. At present, more and more patients with severe knee osteoarthritis have undergone surgical treatment of artificial knee joint replacement. Over the past 20 years, the prognosis and prosthesis survival rate of patients after TKA have been significantly improved with the advancement of prosthesis design and surgical techniques. However, 11.7\% of these patients still have no obvious improvement in clinical efficacy after 1 year, and $10 \%$ to $34 \%$ of the patients 
have long-term postoperative pain. Foreign studies have shown that patient satisfaction after TKA was $81.2 \%$ between 1990 and 1999, and only $85 \%$ between 2000 and 2012 , there were about $22 \%$ of the patients with TKA who gave an "excellent" evaluation. In order to maximize the recovery of knee function, postoperative scientific, systematic, safe and effective rehabilitation is also very important in addition to precise surgical options. However, the psychological rehabilitation, pain rehabilitation, functional exercise, and rehabilitation assessment of patients after TKA have not been fully carried out in China, and they are still in the exploration stage. Patients with knee osteoarthritis often have complications such as diabetes and hypertension, which makes the rehabilitation of TKA difficult. At present, the fact that the total number of diabetic patients in China has ranked the first in the world is indisputable. The fifth edition of the International Diabetes Federation points out that there are more than 92.4 million diabetic patients in China's 20-79 age group, accounting for about a quarter of the total number of diabetic patients worldwide. In 2010, there were more than 90,000 people who were reported to have diabetes and these people were aged 18 in 31 provinces. The data showed that cities the prevalence of diabetes has reached $9.65 \%$.

And all the data show that the prevalence of diabetes in China has increased year by year, especially in recent years, the prevalence rate has risen sharply, and the development trend is not optimistic. With the aging of the population, there are more and more patients with diabetic knee osteoarthritis. At the same time, more and more clinical studies and animal in vivo studies have proved that diabetes is closely related to the occurrence and development of knee osteoarthritis, clearly indicating that diabetes is an independent risk factor for the development of knee osteoarthritis, affecting the therapeutic effect of knee osteoarthritis. Tuomas et al. found that 193 patients who underwent hip and knee replacement had a highest rate of joint pain after surgery, and the multivariate regression curve showed that diabetes was closely related to postoperative pain. In addition, more studies have shown that patients with diabetes require a longer functional recovery period after hip and knee arthroplasty, and functional scores are often lower than non-diabetic patients, thus reducing postoperative satisfaction and quality of life. At the same time, diabetes can also cause a variety of surgical complications, such as poor healing of incisional fat liquefaction [12], increased perioperative bleeding, cardiovascular accidents, deep vein thrombosis of the lower extremities, loosening of the prosthesis, and even mortality. The most common complication is the increase in the risk of postoperative infection, which leads to a significant increase in the rate of joint revision. The main reason is that diabetic patients often have peripheral vascular neuropathy. So, their new capillary growth is slow, local blood supply to the incision is insufficient, nutrients and oxygen transport disorders. At the same time, the protein metabolism in the body is abnormal, and the synthesis and decomposition are unbalanced, resulting in decreased growth of fibroblasts and decreased synthesis of collagen fibers. Moreover, the bone and mineral metabolism of diabetic patients is quite different from normal, and it is easy to be associated with osteoporosis, which further affects the healing of bone. Environmental metabolic disorders in diabetic patients also increase the risk of surgery. Therefore, the rehabilitation of patients with diabetic knee osteoarthritis after TKA is more urgent to solve.

With the development of total knee arthroplasty, postoperative [13] and nursing are receiving more and more attention, which is the key to patients' ability to obtain independent living. Only when surgery and rehabilitation are closely combined can the knee function be restored to the maximum extent, and the patient can return to society and return to work. However, there are still many problems in the current rehabilitation of TKA. First of all, with the reform of rehabilitation methods, the cost of rehabilitation has also increased. Gao Tong and other scholars found that if their departments paid attention to the rehabilitation of TKA in 1997 and improved the training methods and increased the training level of patients, the activity of patients after discharge was obviously improved, but the rehabilitation cost had increased significantly. In addition, TKA surgery has larger wounds, more oozing, extensive osteotomy and soft tissue dissection, and opening of the bone marrow cavity. So, it requires postoperative anticoagulant drugs and early rehabilitation exercises. If the swelling is serious, it can directly affect the blood circulation of the limbs, and even complications such as skin blisters and deep vein thrombosis which have a great influence on TKA. In addition, pain is the biggest problem faced by patients after TKA, and even many patients will reduce or refuse exercise due to pain, affecting the effect of rehabilitation. Foreign scholar Beswick pointed out that $19 \%$ to $31 \%$ of patients with TKA still have rehabilitation effects due to pain. Some studies have also found that, most patients were afraid of continuous passive movement when they used the continuous passive motion (CPM). This phenomenon was expressed as to fear of pain, doubt whether they can withstand, and not want to cooperate with functional exercise. Pain is an important factor affecting the compliance of early functional exercise in patients. The compliance of early rehabilitation exercise decreases with the increase of pain [14]. At present, multi-mode analgesia is used to improve the satisfaction of TKA, effectively reduce postoperative pain and adverse reactions, and promote postoperative recovery. However, excessive use of anesthetic drugs to control pain after surgery may cause nausea and vomiting, respiratory depression, and mental state change and other adverse reactions. In addition to anesthesia and analgesics, there are few low-cost functional rehabilitation methods that can effectively reduce postoperative pain.

Manual lymphatic drainage (MLD) is a special technique. Unlike traditional massage, MLD must be pressed slowly along the lymphatic flow. It is a technique for excreting body fluid toxins. MLD has many benefits, for example: (1) to stimulate the lymphatic system by accelerating lymphatic circulation; (2) to accelerate the discharge of body fluid toxins; 
(3) to strengthen the body's fluid dynamic system, thereby reducing edema; (4) to weaken the sympathetic response and enhance the sensitivity of parasympathetic nerves. MLD is widely used in developed countries such as Germany and the United States and is relatively rare in China. Moiski $\mathrm{P}$ has done a randomized controlled trial in which 20 patients scheduled to undergo surgery for deep venous thrombosis of the lower extremities were randomized into a treatment group and a control group. Patients in the treatment group were treated with MLD for 5 weeks before surgery and the group did not receive special treatment. The VRI, CEAP score and quality of life of the treatment group were significantly improved compared with the control group $(\mathrm{P}<0.05)$. The postoperative pain and swelling of the treatment group were significantly better than the control group, confirming that MLD can be used as Adjunctive treatment of deep venous thrombosis of the lower extremities. Bertelli DF randomly assigned 45 patients with lower extremity edema after bariatric surgery into experimental group and control group. The experimental group was treated with MLD based on routine functional exercise, and the lower extremity volume was observed 1 day and 3 days after operation. The changes showed that the experimental group had a significant reduction in the lower extremity volume relative to the control group, confirming that MLD can effectively alleviate the symptoms of lower extremity edema after bariatric surgery. In a randomized controlled trial, Shan Dayan and others randomly divided 72 patients with upper extremity edema after breast cancer surgery into the experimental group and the control group. The treatment group was treated with MLD on the basis of conventional treatment. After 3 weeks, the results showed that the total effective rate of the treatment group was $83.3 \%$, which was significantly higher than that of the control group (69.4\%). It was confirmed that MLD has a clear clinical effect on upper limb lymphedema after breast cancer operation.

Foreign scholars Claude Pichonnaz and others applied MLD to the rehabilitation of total knee arthroplasty. The treatment group was treated with MLD and the control group received routine treatment. The records were compared before and 2 days, 7 days, and 3 days after surgery. The knee joint activity, swelling degree, pain score, gait and other results of the two groups of patients showed that MLD can relieve early postoperative pain. The results of this study are basically consistent with our study. Another scholar, Ebert, also designed a similar experiment, applying MLD to the rehabilitation of total knee arthroplasty, and recording the two groups of patients 2, 3, 4, and 6 weeks after surgery. Knee joint mobility [15], swelling degree, pain score and other results showed that the knee flexion activity was statistically different only in the two groups at 6 weeks after surgery, but the follow-up time of the study was too short and too scattered, and the experimental results were insufficiently convincing.

\section{Conclusion}

According to the above clinical data, we can get a lot of conclusions. Manual lymphatic drainage therapy can effectively relieve postoperative knee swelling and pain in patients with diabetic knee osteoarthritis after total knee arthroplasty. And the therapy can also accelerate knee joint function recovery, improve postoperative patient satisfaction, and has a clear effect on promoting postoperative functional rehabilitation [16] of diabetic patients.

\section{References}

[1] Yang Z, Reed $\mathrm{T}$ and Longino BH. Bone Scintigraphy SPECT/CT Evaluation of Mandibular Condylar Hyperplasia. J Nucl Med Technol 2016; 44: 49-51.

[2] Edwards PK, Milles JL, Stambough JB, Barnes CL and Mears SC. Inpatient versus Outpatient Total Knee Arthroplasty. J Knee Surg 2019.

[3] Su CA, Copp JA, Weinberg DS, Kraay MJ, Fitzgerald SJ and Wera GD. Are Readmissions After Total Knee Arthroplasty Preventable? J Knee Surg 2019.

[4] Mandell JB, Orr S, Koch J, Nourie B, Ma D, Bonar DD, Shah N and Urish KL. Large variations in clinical antibiotic activity against Staphylococcus aureus biofilms of periprosthetic joint infection isolates. J Orthop Res 2019.

[5] Hiemstra LA, Kerslake S, Kupfer N and Lafave MR. Generalized joint hypermobility does not influence clinical outcomes following isolated MPFL reconstruction for patellofemoral instability. Knee Surg Sports Traumatol Arthrosc 2019.

[6] Yoshimoto K, Noguchi M, Yamada A and Nasu Y. Compensatory Function of the Subtalar Joint for Lower Extremity Malalignment. Adv Orthop 2019; 2019: 7656878.

[7] Ozkuk K and Uysal B. Is the Duration of Spa Cure Treatment Important in Knee Osteoarthritis? A Randomized Controlled Study. Complement Med Res 2019; 1-7.

[8] Yao YY, Zhou QH, Yu LN and Yan M. Additional femoral nerve block analgesia does not reduce the chronic pain after total knee arthroplasty: A retrospective study in patients with knee osteoarthritis. Medicine (Baltimore) 2019; 98: e14991.

[9] Guerero RM, das Neves LMS, Guirro RRJ and Guirro ECO. Manual Lymphatic Drainage in Blood Circulation of Upper Limb With Lymphedema After Breast Cancer Surgery. J Manipulative Physiol Ther 2017; 40: 246-249.

[10] Majewski-Schrage $T$ and Snyder K. The Effectiveness of Manual Lymphatic Drainage in Patients With Orthopedic Injuries. J Sport Rehabil 2016; 25: 91-97.

[11] Veronese N, Stubbs B, Koyanagi A, Vaona A, Demurtas J, Schofield P and Maggi S. Mitochondrial genetic haplogroups and cardiovascular diseases: Data from the Osteoarthritis Initiative. PLoS One 2019; 14: e0213656.

[12] Yang Z, Li YQ, Tang Y, Chen W, Li Q, Zhou CD, Zhao MX and $\mathrm{Hu} \mathrm{CM}$. [Penile augmentation and elongation using autologous dermal-fat strip grafting]. Zhonghua Zheng Xing Wai Ke Za Zhi 2012; 28: 172-176.

[13] Nguyen C, Lefevre-Colau MM, Poiraudeau S and Rannou F. Rehabilitation (exercise and strength training) and osteoarthritis: A critical narrative review. Ann Phys Rehabil Med 2016; 59: 190-195. 
[14] Nieves-Plaza M, Castro-Santana LE, Font YM, Mayor AM and Vila LM. Association of hand or knee osteoarthritis with diabetes mellitus in a population of Hispanics from Puerto Rico. J Clin Rheumatol 2013; 19: 1-6.

[15] Tittinger T, Sloniak R, Szczepanski D, Gazdzik TS, Kulesa-Mrowiecka M and Kikowski L. Lateral instability of the knee joint and disorder of the ankle joint extension disorder in men. Wiad Lek 2019; 72: 250-254.
[16] Van Den Houte M, Van Oudenhove L, Bogaerts K, Van Diest I, De Bie J, Persoons P and Van den Bergh O. Relationship between different experimental measures of distorted symptom perception in functional syndrome patients. Psychosom Med 2019. 